\title{
Prediction of central lymph node metastasis in papillary thyroid microcarcinoma according to clinicopathologic factors and thyroid nodule sonographic features: a case-control study
}

This article was published in the following Dove Press journal: Cancer Management and Research

\author{
Wen-Xu Jin ${ }^{1,2, *}$ \\ Dan-Rong $\mathrm{Ye}^{2, *}$ \\ Yi-Han Sun ${ }^{2, *}$ \\ Xiao-Fen Zhou ${ }^{2}$ \\ Ou-Chen Wang ${ }^{2}$ \\ Xiao-Hua Zhang ${ }^{2}$ \\ Ye-Feng Cai ${ }^{2}$
}

'Department of Vascular Surgery, The First Affiliated Hospital of Wenzhou Medical University, Wenzhou, Zhejiang Province, 325000, China; ${ }^{2}$ Department of Breast and Thyroid Surgery, The First Affiliated Hospital of Wenzhou Medical University, Wenzhou, Zhejiang Province, 325000, China

*These authors contributed equally to this work

Correspondence: Ye-Feng Cai; Xiao-Hua Zhang

Department of Breast and Thyroid Surgery, The First Affiliated Hospital of Wenzhou Medical University, Nanbai

Xiang, Ouhai, Wenzhou, Zhejiang

Province 325000 , China

$\mathrm{Tel} / \mathrm{Fax}+8657755579462$

Email zxhoncology0577@sina.com;

cyfoncology@gmail.com
Background: Preoperative diagnosis of central lymph node metastasis (CLNM) poses to be a challenge in clinical node-negative papillary thyroid microcarcinoma (PTMC). This research work aims at investigating the association existing between BRAF mutation, clinicopathological factors, ultrasound characteristics, and CLNM, in addition to establishing a predictive model for CLNM in PTMC.

Materials and methods: The study included 673 PTMC patients, already undergone total thyroidectomy or lobectomy with prophylactic central lymph node dissection. The predictor factors were identified through univariate and multivariate analyses. The support vector machine was put to use to develop statistical models, which could predict CLNM on the basis of independent predictors.

Results: Tumor size ( $>5 \mathrm{~mm}$ ), lower location, no well-defined margin, contact of $>25 \%$ with the adjacent capsule, display of enlarged lymph nodes, and BRAF mutation were independent predictors of CLNM. Through the use of the predictive model, $79.6 \%$ of the patients were classified accurately, the sensitivity and specificity amounted to be $85.1 \%$ and $75.8 \%$, respectively, and the positive predictive value and negative predictive value stood at $71.6 \%$ and $87.6 \%$, respectively. Conclusions: We established a predictive model in order to predict CLNM preoperatively in PTMC when preoperative diagnosis of CLNM was not clear.

Keywords: papillary thyroid microcarcinoma, central lymph node, predictive factor, support vector machine

\section{Background}

Thyroid cancer (TC) was the most frequently found endocrine malignancy. ${ }^{1}$ As of now, the incidence of TC had been experiencing a growing trend throughout the past era, being faster in comparison with any other cancer type in the USA. ${ }^{2,3}$ Among these patients, papillary thyroid cancer (PTC) appeared to be the most widespread histological TC. ${ }^{3}$ The definition of the papillary thyroid microcarcinoma (PTMC) was made as a tumor with a maximum diameter of $\leq 1.0 \mathrm{~cm},{ }^{4}$ and this proportion had increased over time because of the utilization of sonography in addition to ultrasound (US)-guided fine-needle aspiration biopsy (FNAB); up to $50 \%$ of newly diagnosed PTC were in the form of PTMC. ${ }^{5,6}$ Even though most of the patients having PTMC possessed an indolent clinical course and positive prognosis, ${ }^{7}$ yet they usually had lymph node metastasis when diagnosed. ${ }^{8}$ Central lymph node metastasis (CLNM) had been taken into consideration being an element of risk regarding the recurrence, 
distant metastasis, and reduced survival. ${ }^{9-11}$ Lateral lymph node metastasis was normally via central compartment. ${ }^{12}$ Therefore, it was crucial to find the elements of risk predicting CLNM in PTMC.

BRAF mutation was the most commonly found genetic alteration in TC, taking place in approximately $45 \%$ of sporadic $\mathrm{PTCs}$ in addition to being a major reason giving rise to atypical trigger of the MAPK pathway in human cancers. ${ }^{13}$ The T1799A BRAF mutation gives birth to a V600E amino acid variation in the BRAF protein and occurs uniquely in PTC as well as some PTC-derived anaplastic TCs. ${ }^{14}$ This mutation was associated with clinicopathological characteristics that relate to tumor progression and recurrence such as extrathyroidal invasion, lymph node metastasis, and advanced tumor stages. ${ }^{15}$

It was brought to light by the past reports on PTMC that aggressive characteristics and poor outcomes had association with US characteristics. ${ }^{16-18}$ In this study, we investigated the association between BRAF mutation, clinicopathological factors, US characteristics, and CLNM in PTMC. Furthermore, we also developed a predictive model for the purpose of predicting preoperative CLNM in PTMC.

\section{Materials and methods}

\section{Patients}

The study included 673 PTMC patients who had undergone total thyroidectomy or lobectomy with prophylactic central lymph node dissection (pCLND) at the First Affiliated Hospital of Wenzhou Medical University from March 2016 to March 2017. All pathological diagnoses were made by three experienced pathologists. Preoperative US-guided FNAB was also carried out on the primary thyroid tumor in each patient suspected of malignancy. The PTMC patients included 147 males and 526 females. All patients had no history of neck surgery or neck irradiation. Retrospective reviews of electronic clinical and pathologic records were carried out to collect the clinicopathologic information. Approval of the research protocol was received from the Ethics Committee of the First Affiliated Hospital of Wenzhou Medical University.

\section{Imaging analysis}

The performance of US was made with the help of Acuson Sequoia and 128XP sonographic scanners (Siemens Medical Solutions, Mountain View, CA, USA) that were equipped with 8-13 MHz linear probes. The factors selected to analyze the risk of CLNM are shown in Table 1. Assessment of the tumor size was done by maximum diameter based on US.
The definition of Multifocal PTMC was provided as being above one lesion in a paraffin section, and the lesion with the maximum diameter or the most doubtful dominant nodule was brought under analysis. Shape was divided into regular or irregular. Classification of internal composition was made as either totally solid or largely solid (proportion of solid components $\geq 50 \%$ ) cystic. Classification of margin was made as well defined and not well defined. On the basis of preoperative US images, classification of tumor position was made in four areas (upper, middle, lower, and isthmus). Contact of thyroid mass with the thyroid capsule as seen on US was recorded, and $>25 \%$ contact with the adjacent capsule is the most accurate measurement for predicting extrathyroidal extension. ${ }^{18}$

\section{DNA extraction and ARMS PCR}

Two or three US-guided fine-needle aspiration using a 25-guage needle was carried out for the purpose of collecting material for both the cytological and the molecular analyses. The extraction of the DNA from the FNAB specimens was performed with the use of the DNA Extraction Kit (Amoy Diagnostics, Xiamen, China) in accordance with the guidelines of the manufacturer. Human BRAFV600E ARMS-PCR Kit (Amoy Diagnostics, Xiamen, China) based on Amplification Refractory Mutation System (ARMS) was used for the detection of BRAFV600E mutation (approved by China Food and Drug Administration [CFDA]). Every sample was subjected to an external control assay and a mutations assay in the same well. Each test included a positive control and a negative control. BRAFV600E mutation analysis was carried out on the $\mathrm{ABI}$ prism 7,500 series real-time polymerase chain reaction (PCR) instrument (Applied Biosystems, Carlsband, CA, USA). PCR procedure was as follows: Stage I: 5 minutes, $95^{\circ} \mathrm{C}$ for 1 cycle; Stage $2: 25$ seconds, $95^{\circ} \mathrm{C}$, 20 seconds, $64^{\circ} \mathrm{C}, 20$ seconds, $72^{\circ} \mathrm{C}$, for 15 cycles; Stage 3 : 25 seconds, $93^{\circ} \mathrm{C}, 35$ seconds, $60^{\circ} \mathrm{C}, 20$ seconds, $72^{\circ} \mathrm{C}$ for 31 cycles (Data Collection).

\section{Model development}

The support vector machine (SVM) was put to use in respect of the development of statistical models that could predict CLNM based on BRAF mutation, clinicopathological factors, and US characteristics. We established the SVM model by utilizing Libsvm 3.20 for the prediction of CLNM with the modeling platform of MATLAB 2014a. The C-SVC together with radial basis function kernel was chosen. Moreover, the grid search approach was employed for debugging the model during the development of the SVM model. The grid c stood from -8 to 8 , the grid c step amount to be 0.5 , the grid $g$ bound 
Table I Baseline characteristics of papillary thyroid microcarcinoma patients stratified by the status of metastatic central lymph nodes

\begin{tabular}{|c|c|c|c|}
\hline \multirow[t]{2}{*}{ Characteristics } & \multicolumn{2}{|c|}{ The status of metastatic central lymph nodes } & \multirow[t]{2}{*}{$P$-value } \\
\hline & Positive & Negative & \\
\hline$\overline{T \text { Total }}$ & $28 I(4 I .8 \%)$ & $392(58.2 \%)$ & \\
\hline Tumor size (mm) & $7.11 \pm 1.90$ & $6.44 \pm 1.81$ & $<0.001$ \\
\hline$\leq 5(n, \%)$ & $68(24.2 \%)$ & $123(3 \mid .4 \%)$ & 0.042 \\
\hline$>5(n, \%)$ & $213(75.8 \%)$ & $269(68.6 \%)$ & \\
\hline Age at diagnosis (years) & $46.37 \pm 10.44$ & $47.58 \pm 10.44$ & 0.14 \\
\hline$<45(\mathrm{n}, \%)$ & $12 \mid(43.1 \%)$ & $152(38.8 \%)$ & 0.264 \\
\hline$\geq 45(\mathrm{n}, \%)$ & $160(56.9 \%)$ & $240(61.2 \%)$ & \\
\hline Gender & & & 0.13 \\
\hline Male (n, \%) & $82(29.2 \%)$ & $94(24.0 \%)$ & \\
\hline Female (n, \%) & $199(70.8 \%)$ & $298(76.0 \%)$ & \\
\hline BRAF mutation & & & $<0.001$ \\
\hline Yes (n, \%) & $243(86.5 \%)$ & $294(75.0 \%)$ & \\
\hline No $(n, \%)$ & $38(13.5 \%)$ & $98(25.0 \%)$ & \\
\hline Tumor location & & & $<0.001$ \\
\hline Lower (n, \%) & $78(27.8 \%)$ & $61(15.6 \%)$ & \\
\hline Upper/middle/isthmus (n, \%) & $203(72.2 \%)$ & $331(84.4 \%)$ & \\
\hline Margin & & & 0.002 \\
\hline Well defined (n, \%) & $167(59.4 \%)$ & $278(70.9 \%)$ & \\
\hline Not well defined $(n, \%)$ & II 4 (40.6\%) & II 14 (29.1\%) & \\
\hline Contact of $>25 \%$ with the adjacent capsule & & & $<0.001$ \\
\hline Yes (n, \%) & $159(56.6 \%)$ & 55 (I5.2\%) & \\
\hline No $(n, \%)$ & $122(43.4 \%)$ & $337(84.8 \%)$ & \\
\hline Display of enlarged lymph nodes & & & $<0.001$ \\
\hline Present (n, \%) & 157 (55.9\%) & 127 (32.4\%) & \\
\hline Absent (n, \%) & $124(44.1 \%)$ & $265(67.6 \%)$ & \\
\hline No. of nodules & & & 0.117 \\
\hline Single (n, \%) & $221(78.6 \%)$ & $327(83.4 \%)$ & \\
\hline Multiple (n, \%) & $60(21.4 \%)$ & $65(16.6 \%)$ & \\
\hline Hashimoto's thyroiditis & & & 0.262 \\
\hline Yes $(n, \%)$ & $57(20.3 \%)$ & 95 (24.2\%) & \\
\hline No $(n, \%)$ & 224 (79.7\%) & $297(75.8 \%)$ & \\
\hline Calcification & & & 0.001 \\
\hline Present (n, \%) & 175 (62.2\%) & 194 (49.5\%) & \\
\hline Absent (n, \%) & $106(37.8 \%)$ & $198(50.5 \%)$ & \\
\hline Shape & & & 0.659 \\
\hline Regular (n, \%) & $239(85.1 \%)$ & 337 (86.0\%) & \\
\hline Irregular (n, \%) & 42 (I4.9\%) & 55 (I4.0\%) & \\
\hline Intact capsule & & & 0.480 \\
\hline Yes (n, \%) & $152(54.1 \%)$ & $223(56.9 \%)$ & \\
\hline No $(n, \%)$ & $129(45.9 \%)$ & $169(43.1 \%)$ & \\
\hline Composition & & & 0.793 \\
\hline Solid (n, \%) & 274 (97.5\%) & 384 (98.0\%) & \\
\hline Cystic or mixed $(n, \%)$ & $7(2.5 \%)$ & $8(2.0 \%)$ & \\
\hline Vascularization & & & 0.506 \\
\hline Present (n, \%) & 14 (5.0\%) & $25(6.4 \%)$ & \\
\hline Absent (n, \%) & $267(95.0 \%)$ & $367(93.6 \%)$ & \\
\hline
\end{tabular}

value came out to be -8 to 8 , and the grid $g$ step valued at 0.5 . The fold number of cross-validation came out to be 5 .

$$
\text { Plabel }=\operatorname{sgn}\left(\sum_{\mathrm{i}=0}^{\mathrm{n}} \mathrm{w}_{\mathrm{i}} \exp \left(-\operatorname{gamma}\left|\left(\mathrm{x}_{\mathrm{i}}-\mathrm{x}\right)\right|^{2}+\mathrm{b}\right)\right)
$$

The "sgn" function is to maintain the positive or negative of the parameter. The meaning of "plabel" is to get the numeric symbols. As we divided patients into two groups, positive value means central lymph node metastasis positive and negative value means central lymph node metastasis negative.

\section{Statistical analysis}

We created two groups of the patients in accordance with the status of metastatic central lymph node: CLNM positive and 
CLNM negative. The application of independent two-sample $t$-test was made in order to carry out the comparison of data on normal distribution. Categorical variables were expressed as percentage and were compared with chi-squared test or Fisher's exact test, as appropriate. Variables with $P<0.05$ in the univariate analysis were progressed to a multivariate analysis using forward stepwise selection. The findings were brought forth as odds ratio (OR) having 95\% CI as well as $P$-value. All $P$-values were two sided. Moreover, the value $<0.05$ was taken into consideration as statistically significant. Statistical analysis was performed with SPSS software version 22.0 (SPSS, Chicago, IL, USA).

\section{Ethics approval and informed consent}

The research protocol used in this study was approved by the Ethics Committee of the First Affiliated Hospital of Wenzhou Medical University. All of the patients provided written informed consent.

\section{Results}

\section{Relationship of central lymph} node metastasis of PTMC with clinicopathologic factors and US features

We carried out the investigation of the relationship of CLNM with clinicopathologic factors and US features through univariate analysis (Table 1). The results showed that larger tumor size $(P<0.001)$, BRAF mutation $(P<0.001)$, lower location $(P<0.001)$, presence of calcification $(P<0.001)$, no well-defined margin $(P=0.002)$, contact of $>25 \%$ with the adjacent capsule $(P<0.001)$, and display of enlarged lymph nodes $(P<0.001)$ have considerable association with CLNM.

The use of multivariate logistic regression was made for the purpose of determining whether these elements were independently associated with CLNM (Table 2). The results showed that larger tumor (OR 1.164; $P=0.005 ; 95 \% \mathrm{CI}$ 1.048-1.294), lower location (OR 1.912; $P=0.006 ; 95 \% \mathrm{CI}$ 1.206-3.034), no well-defined margin (OR 1.695; $P=0.010$;

Table 2 Multivariate analysis between the clinicopathologic factors and CLNM

\begin{tabular}{llll}
\hline Characteristic & OR & $\mathbf{9 5 \%} \mathbf{~ C l}$ & P-value \\
\hline Tumor size $(\mathrm{mm})$ & $\mathrm{I} .164$ & $\mathrm{I} .048-1.294$ & 0.005 \\
Tumor location & $\mathrm{I} .912$ & $\mathrm{I} .206-3.034$ & 0.006 \\
Margin & $\mathrm{I} .695$ & $\mathrm{I} .136-2.530$ & 0.010 \\
Display of enlarged lymph nodes & 5.865 & $3.786-9.087$ & $<0.00 \mathrm{I}$ \\
BRAF mutation & 2.290 & $1.404-3.734$ & $0.00 \mathrm{I}$ \\
Contact of $>25 \%$ with the & 13.833 & $8.696-22.007$ & $<0.00 \mathrm{I}$ \\
adjacent capsule & & & \\
\hline
\end{tabular}

Abbreviations: CLNM, central lymph node metastasis; OR, odds ratio.
95\% CI 1.136-2.530), contact of $>25 \%$ with the adjacent capsule (OR 13.833; $P<0.001 ; 95 \%$ CI 8.696-22.007), display of enlarged lymph nodes (OR 5.865; $P<0.001 ; 95 \% \mathrm{CI}$ 3.786-9.087), and BRAF mutation (OR 2.290; $P=0.001$; 95\% CI 1.404-3.734) were independently predictive of CLNM.

\section{The predictive model}

An aggregate of six selected factors was put into use to predict CLNM in 673 patients. The details of patients are shown in Table 1. We established a model for predicting CLNM using SVM statistical model that combines the six factors. Five hundred thirty-six of 673 patients $(79.6 \%)$ were correctly classified and the details are shown in Table 3 . The sensitivity and specificity for this model amounted to $85.1 \%$ and $75.8 \%$, respectively, and the positive predictive value and negative predictive value stood at $71.6 \%$ and $87.6 \%$, respectively (Table 4). The model had a satisfied predictive value when compared with other single predictors.

\section{Discussion}

In the current era, the prevalence of PTMC has experienced a rapid growth across the globe through preoperative sonography and US-guided FNAB. Despite the fact that most of the patients having PTMC had an indolent clinical course together with positive prognosis, the incidence of CLNM has been termed as an element of risk with respect to poor prognosis. ${ }^{9-11}$ pCLND is brought forth as a suggestion in respect of the decrease of recurrence and metastasis; however, aggressive PTMC treatments can lead to negative results, for instance, temporary or permanent laryngeal nerve injury causing damage to the voice and temporary or permanent hypoparathyroidism. ${ }^{19,20}$ Some studies reported that aggressive treatments were not linked to the improved outcome as regards reduced recurrence or prolonged survival. ${ }^{9}$ That is why monitoring could be chosen for the patients with PTMC without unfavorable features. ${ }^{7}$ Although the prognosis of most PTMC appears to be positive, a small part of the patients having PTMC has a more aggressive course of disease. ${ }^{21}$ However, the decision of surgery is dependent on the opinion

Table 3 The predictive model and pathological diagnosis of CLNM

\begin{tabular}{llll}
\hline Pathological & \multicolumn{3}{l}{ Predictive model } \\
\cline { 2 - 4 } diagnosis & Positive & Negative & Total \\
\hline Positive & 239 & 42 & 281 \\
Negative & 95 & 297 & 392 \\
Total & 334 & 339 & 673 \\
\hline
\end{tabular}

Abbreviation: CLNM, central lymph node metastasis. 
Table 4 The predictive value for the model and independent predictors

\begin{tabular}{lllll}
\hline Independent predictors & Sensitivity (\%) & Specificity (\%) & PPV (\%) & NPV (\%) \\
\hline Size & 75.8 & 31.9 & 79.8 & 64.8 \\
Location & 27.8 & 84.4 & 56.1 & 62.0 \\
Margin & 40.6 & 70.9 & 50.0 & 62.5 \\
Display of enlarged lymph nodes & 55.9 & 67.6 & 55.3 & 68.1 \\
BRAF mutation & 86.5 & 25.0 & 45.3 & 72.1 \\
Contact of $>$ 25\% with the adjacent capsule & 56.6 & 86.0 & 74.3 & 73.4 \\
The model & 85.1 & 75.8 & 71.6 & 87.6 \\
\hline
\end{tabular}

Abbreviations: NPV, negative predictive value; PPV, positive predictive value.

of the doctor or the patients. In this study, we set an aim to dig deep into the preoperative predicting elements for CLNM, together with allowing the surgeons and endocrinologists to formulate a more comprehensive operative plan.

In respect of this research work, the occurrence rate of CLNM in PTMC came out to be $41.8 \%$ that displayed harmony with the previous reports. ${ }^{11,22}$ It was illustrated by the univariate analysis that larger tumor size, BRAF mutation, lower location, the presence of calcification, no well-defined margin, contact of $>25 \%$ with the adjacent capsule, and display of enlarged lymph nodes were considerably linked to the status of CLNM. Multivariate logistic regression confirmed that larger tumor size, BRAF mutation, lower location, no well-defined margin, contact of $>25 \%$ with the adjacent capsule, and display of enlarged lymph nodes were independently predictive of CLNM.

In our study, cytological specimens from US-guided fineneedle aspiration were used to detect BRAF mutation and the occurrence of the BRAF mutation at $79.8 \%$ (537/673), which was higher than generally reported values of approximately $45 \%-50 \% .^{23,24}$ One possible explanation suggests that the technique of ARMS-PCR had a higher sensitivity and specificity. ${ }^{25}$ Another reason was that elevated consumption of iodine poses to be a sizeable element of risk with respect to the incidence of BRAF mutation. ${ }^{26}$ Taking into consideration the eastern coastal regions in China, where the study was conducted, the population's diet is rich in iodine.

With the increase in size, lymph node metastasis increased in PTMC. ${ }^{27}$ Several studies revealed that larger tumor size was an independent predictor of CLNM. ${ }^{28,29}$ Kim et al ${ }^{28}$ discovered that tumor size $>5 \mathrm{~mm}$ in PTMC was sizably associated with the CLNM. Our research work brought to light the fact that tumor size $>5 \mathrm{~mm}$ was independently predictive of CLNM in PTMC.

Some studies had reported that the incidence of CLNM was related to the location of PTMC. ${ }^{11}$ Lower location in the thyroid lobe suggested an elevated risk factor of CLNM. ${ }^{30}$ In the same way with previous studies, we discovered the fact that the tumor positioned in the lower pole of the thyroid was an independent factor associated with CLNM.
The thyroid nodule with no well-defined margin is more inclined to be malignant tumor. It was reported that no well-defined margin suggested a considerable US feature of aggressive PTMC. ${ }^{17}$ Furthermore, we also discovered that no well-defined margin suggested an independent predictor of CLNM in PTMC.

Contact with $>25 \%$ of the capsule is the most accurate measurement for predicting extrathyroidal extension. ${ }^{18}$ It was reported by the past studies that extrathyroidal extension was independently predictive of CLNM in patients having PTMC. ${ }^{27,31}$ In our study, we discovered the fact that thyroid nodules in contact with $>25 \%$ of the capsule were an independent factor in respect of CLNM.

Enlarged lymph nodes are usually associated with lymph node metastasis and recur in the regional lymph nodes. ${ }^{32}$ In our study, display of enlarged lymph nodes suggested an independent predictor of CLNM in accordance with the univariate and multivariate analyses.

The incidence of recurrence, distant metastasis, and reduced survival ${ }^{9-11}$ increased with the existence of CLNM in PTMC. pCLND still continued to be a point that needs discussion. The CLND could be carried out intraoperatively if the CLNM could be diagnosed preoperatively. It had been reported by numerous studies that BRAF mutation, ${ }^{13-15}$ overexpression of cyclin D1, underexpression of $\mathrm{p} 27^{33}$ together with other PTC-specific mutations, are likely to be linked with the aggressiveness of thyroid carcinoma. Moreover, the BRAF mutation was the most frequent somatic oncogene in PTC. ${ }^{34}$ Owing to the fact that preoperative sonography for the detection of CLNM possessed low sensitivity, we employed a combination of BRAF mutation, clinicopathologic features, and US findings for the purpose of enhancing the diagnostic accuracy. Enlightened by a US scoring system in predicting CLNM ${ }^{35}$ we developed a model based on six factors. This model for the detection of CLNM had a sensitivity of $85.1 \%$, a specificity of $75.8 \%$, a positive predictive value of $71.6 \%$, and a negative predictive value of $87.6 \%$. Moreover, the model is expected to extend help to the surgeons when it comes to deciding the surgical strategy with respect to each patient in PTMC. 
There were numerous constraints encountered by the current research work. Firstly, in some patients having multifocal nodules, we carried out analysis of the most suspicious or the largest one only, when the information about other nodules was not available. Secondly, the model has characterized a reference set, which needs more patients to examine the characteristics predicting CLN metastatic disease in a test set. Thirdly, there is a need for an extended follow-up time for the purpose of evaluating the survival rate as well as surgical complication. In summary, we discovered six independent factors, tumor size ( $>5 \mathrm{~mm}$ ), lower location, no well-defined margin, contact of $>25 \%$ with the adjacent capsule, display of enlarged lymph nodes, and BRAF mutation, being the independent predictors of CLNM. Thereafter, we established a predictive model with the use of a simple approach in order to predict CLNM preoperatively in PTMC as preoperative diagnosis of CLNM was not quite clear enough.

\section{Acknowledgments}

This work was supported by a grant from Major Science and Technology Projects of Zhejiang Province (NO. 2015C03052), Wenzhou Science and Technology Planning Project (NO Y20160126, 2017Y0929), and Scientific Research Incubator Project of The First Affiliated Hospital of Wenzhou Medical University (NO. FHY2014018).

\section{Disclosure}

The authors report no conflicts of interest in this work.

\section{References}

1. Rahib L, Smith BD, Aizenberg R, Rosenzweig AB, Fleshman JM, Matrisian LM. Projecting cancer incidence and deaths to 2030: the unexpected burden of thyroid, liver, and pancreas cancers in the United States. Cancer Res. 2014;74(11):2913-2921.

2. Chen AY, Jemal A, Ward EM. Increasing incidence of differentiated thyroid cancer in the United States, 1988-2005. Cancer. 2009;115(16):3801-3807.

3. Pellegriti G, Frasca F, Regalbuto C, Squatrito S, Vigneri R. Worldwide increasing incidence of thyroid cancer: update on epidemiology and risk factors. J Cancer Epidemiol. 2013;2013:965212-10.

4. Sobin LH. Histological typing of thyroid tumours. Histopathology. 1990;16(5):513.

5. Hughes DT, Haymart MR, Miller BS, Gauger PG, Doherty GM. The most commonly occurring papillary thyroid cancer in the United States is now a microcarcinoma in a patient older than 45 years. Thyroid. 2011;21(3):231-236.

6. Davies L, Welch HG. Increasing incidence of thyroid cancer in the United States, 1973-2002. Jama. 2006;295(18):2164-2167.

7. Ito Y, Uruno T, Nakano K, et al. An observation trial without surgical treatment in patients with papillary microcarcinoma of the thyroid. Thyroid. 2003;13(4):381-387.

8. Hay ID, Grant CS, van Heerden JA, Goellner JR, Ebersold JR, Bergstralh EJ. Papillary thyroid microcarcinoma: a study of 535 cases observed in a 50-year period. Surgery. 1992;112(6):1139-1146.
9. Hay ID, Hutchinson ME, Gonzalez-Losada T, et al. Papillary thyroid microcarcinoma: a study of 900 cases observed in a 60 -year period. Surgery. 2008;144(6):980-988.

10. Chow SM, Law SC, Chan JK, Au SK, Yau S, Lau WH. Papillary microcarcinoma of the thyroid-Prognostic significance of lymph node metastasis and multifocality. Cancer. 2003;98(1):31-40.

11. Wada N, Duh QY, Sugino K, et al. Lymph node metastasis from 259 papillary thyroid microcarcinomas: frequency, pattern of occurrence and recurrence, and optimal strategy for neck dissection. Ann Surg. 2003;237(3):399-407.

12. Lan X, Sun W, Zhang H, Dong W, Wang Z, Zhang T. A meta-analysis of central lymph node metastasis for predicting lateral involvement in papillary thyroid carcinoma. Otolaryngol Head Neck Surg. 2015;153(5):731-738.

13. Xing M. BRAF mutation in thyroid cancer. Endocr Relat Cancer. 2005;12(2):245-262.

14. Nikiforov YE, Nikiforova MN. Molecular genetics and diagnosis of thyroid cancer. Nat Rev Endocrinol. 2011;7(10):569-580.

15. Xing M. BRAF mutation in papillary thyroid cancer: pathogenic role, molecular bases, and clinical implications. Endocr Rev. 2007;28(7):742-762.

16. Lin KL, Wang OC, Zhang XH, Dai XX, Hu XQ, Qu JM. The BRAF mutation is predictive of aggressive clinicopathological characteristics in papillary thyroid microcarcinoma. Ann Surg Oncol. 2010;17(12):3294-3300.

17. Ito Y, Kobayashi K, Tomoda C, et al. Ill-defined edge on ultrasonographic examination can be a marker of aggressive characteristic of papillary thyroid microcarcinoma. World J Surg. 2005;29(8):1007-1011; discussion 1011-1002.

18. Kwak JY, Kim EK, Youk JH, et al. Extrathyroid extension of welldifferentiated papillary thyroid microcarcinoma on US. Thyroid. 2008;18(6):609-614.

19. Conzo G, Mauriello C, Docimo G, et al. Clinicopathological pattern of lymph node recurrence of papillary thyroid cancer. Implications for surgery. Int J Surg. 2014;12(Supp1 1):S194-S197.

20. Conzo G, Tartaglia E, Avenia N, et al. Role of prophylactic central compartment lymph node dissection in clinically N0 differentiated thyroid cancer patients: analysis of risk factors and review of modern trends. World J Surg Oncol. 2016;14:149.

21. Noguchi S, Yamashita H, Uchino S, Watanabe S. Papillary microcarcinoma. World J Surg. 2008;32(5):747-753.

22. Roh JL, Kim JM, Park CI. Central cervical nodal metastasis from papillary thyroid microcarcinoma: pattern and factors predictive of nodal metastasis. Ann Surg Oncol. 2008;15(9):2482-2486.

23. Tufano RP, Teixeira GV, Bishop J, Carson KA, Xing M. BRAF mutation in papillary thyroid cancer and its value in tailoring initial treatment: a systematic review and meta-analysis. Medicine. 2012;91(5):274-286.

24. Lee JH, Lee ES, Kim YS. Clinicopathologic significance of BRAF V600E mutation in papillary carcinomas of the thyroid: a meta-analysis. Cancer. 2007;110(1):38-46.

25. Huang T, Zhuge J, Zhang WW. Sensitive detection of BRAF V600E mutation by Amplification Refractory Mutation System (ARMS)-PCR. Biomark Res. 2013;1(1):3.

26. Guan H, Ji M, Bao R, et al. Association of high iodine intake with the T1799A BRAF mutation in papillary thyroid cancer. J Clin Endocrinol Metab. 2009;94(5):1612-1617.

27. Zhang L, Wei WJ, Ji QH, et al. Risk factors for neck nodal metastasis in papillary thyroid microcarcinoma: a study of 1066 patients. J Clin Endocrinol Metab. 2012;97(4):1250-1257.

28. Kim KE, Kim EK, Yoon JH, Han KH, Moon HJ, Kwak JY. Preoperative prediction of central lymph node metastasis in thyroid papillary microcarcinoma using clinicopathologic and sonographic features. World J Surg. 2013;37(2):385-391.

29. Lee KJ, Cho YJ, Kim SJ, et al. Analysis of the clinicopathologic features of papillary thyroid microcarcinoma based on 7-mm tumor size. World J Surg. 2011;35(2):318-323.

30. Xu D, Lv X, Wang S, Dai W. Risk factors for predicting central lymph node metastasis in papillary thyroid microcarcinoma. Int J Clin Exp Pathol. 2014;7(9):6199-6205. 
31. So YK, Son YI, Hong SD, et al. Subclinical lymph node metastasis in papillary thyroid microcarcinoma: a study of 551 resections. Surgery. 2010;148(3):526-531.

32. Ito Y, Miyauchi A. A therapeutic strategy for incidentally detected papillary microcarcinoma of the thyroid. Nat Clin Pract Endocrinol Metab. 2007;3(3):240-248.

33. Khoo ML, Beasley NJ, Ezzat S, Freeman JL, Asa SL. Overexpression of cyclin D1 and underexpression of p27 predict lymph node metastases in papillary thyroid carcinoma. J Clin Endocrinol Metab. 2002;87(4):1814-1818.
34. Costa AM, Herrero A, Fresno MF, et al. BRAF mutation associated with other genetic events identifies a subset of aggressive papillary thyroid carcinoma. Clin Endocrinol. 2008;68(4):618-634.

35. Koike E, Noguchi S, Yamashita H, et al. Ultrasonographic characteristics of thyroid nodules: prediction of malignancy. Arch Surg. 2001;136(3):334-337.
Cancer Management and Research

\section{Publish your work in this journal}

Cancer Management and Research is an international, peer-reviewed open access journal focusing on cancer research and the optimal use of preventative and integrated treatment interventions to achieve improved outcomes, enhanced survival and quality of life for the cancer patient The manuscript management system is completely online and includes

\section{Dovepress}

a very quick and fair peer-review system, which is all easy to use. Visit http://www.dovepress.com/testimonials.php to read real quotes from published authors. 